\title{
UTILIZAÇÃO DE ALGORITMOS GENÉTICOS EM METODOLOGIA MULTICRITÉRIO: UMA SOLUÇÃO PARA INCONSISTÊNCIA MATRICIAL
}

\section{UTILIZATION OF GENETIC ALGORITHMS IN MULTICRITERIA METHODOLOGY: A SOLUTION FOR MATRIX INCONSISTENCY}

\author{
José Fabiano da Serra Costa ${ }^{1}$; Augusto José Maurício Wanderley ${ }^{2}$; Carlos Alberto Nunes Cosenza ${ }^{3}$ \\ ${ }^{1}$ Universidade do Estado do Rio de Janeiro - Rio de Janeiro - Brasil - fabiano@ime.uerj.br \\ ${ }^{2}$ Universidade do Estado do Rio de Janeiro - Rio de Janeiro - Brasil - wandmja@momentus.com.br \\ ${ }^{3}$ Universidade do Estado do Rio de Janeiro - Rio de Janeiro - Brasil - cosenza@pep.ufrj.b
}

Recebido para publicação em: 01/06/05

Aceito para publicação em: 18/10/05

\begin{abstract}
Resumo
Este trabalho apresenta uma proposta para solução do problema de inconsistência em matrizes decisórias utilizando algoritmos genéticos. Os Algoritmos Genéticos são modelos computacionais de pesquisa probabilística que imitam a evolução natural das espécies, combinando os conceitos de adaptação seletiva e sobrevivência dos mais capazes. São considerados uma poderosa técnica de otimização estocástica e, provavelmente a mais importante técnica de computação evolucionária. Sua aplicação ao caso de matrizes decisórias permite detectar a inconsistência matricial, enquanto possibilita o fornecimento de soluções alternativas ao tomador de decisão.
\end{abstract}

Palavras-Chave: decisão; inconsistência matricial; algoritmos genéticos.

\section{Introdução}

Sempre que se fala em tomar uma decisão, surge a idéia de algo impreciso, ou indefinido. O ser humano vem se defrontando com este questionamento durante todos os dias, horas, momentos de sua existência, seja através de decisões de caráter individual seja através de decisões coletivas. Se, em eras remotas as conseqüências de uma decisão só afetavam um único ser ou os poucos com que este se relacionava, hoje em dia uma decisão pode afetar uma multidão de outros seres. Os processos de tomada de decisão assumem assim objetivos diversos dentro deste contexto. De qualquer forma, a essência do problema de decisão se resume no fato de não poderem ser alcançados os objetivos (resultados esperados) sem que seja efetuada uma ação, melhor dizendo uma tomada uma decisão.

A partir da idéia de que a tomada de decisão é um processo inevitável, surge então a necessidade de se criar métodos ou formas de facilitar a tomada de decisão. Esses métodos ou metodologias acompanham o ser humano em suas mais variadas atividades, quer sejam profissionais ou não. Com base nestas convicções, foram surgindo diversas alternativas para ajudar o indivíduo (ou organização) a chegar a uma melhor escolha. Nesse contexto, devido à complexidade do ambiente econômico e social e a cadência vertiginosa da inovação tecnológica, particularmente nos domínios da informática e das comunicações, fica claro que o progresso 
depende cada vez mais da adoção de procedimentos de planejamento e gestão inovadores. Surge então a idéia dos chamados métodos de apoio à tomada de decisão.

Esses métodos, conhecidos como Sistemas de Apoio à Decisão (SAD), podem ser definidos como ferramentas geralmente computacionais que envolvem modernas técnicas de sistemas de informação, inteligência artificial, métodos quantitativos de estatística, psicologia cognitiva e comportamental, sociologia das organizações, entre outros, e visam oferecer ao usuário condições favoráveis (suporte) para melhor escolher uma entre diversas alternativas, minimizando assim a chance de erro na tomada de decisão. Dentre os métodos de apoio à decisão, estão as Metodologias Multicritério de Apoio à Decisão (ROY, 1985), com suas diversas escolas, visões e metodologias específicas; as chamadas técnicas de conclaves (ou técnicas de grupo), utilizadas na maioria das vezes quando nos defrontamos com critérios qualitativos (COSENZA e SOUZA, 1996), entre outros. Muitos desses métodos utilizam matrizes decisórias criadas por especialistas para priorizar ou hierarquizar indicadores (ou alternativas - critérios), por exemplo, (WANDERLEY e COSTA, 2002).

Entretanto, pode ocorrer que, com qualquer dos métodos utilizados, a matriz final embora representativa da opinião dos especialistas, seja pouco consistente (respeite muito pouco as propriedades necessárias, tais como transitividade e reciprocidade). Isto pode vir a ocorrer devido a falhas na consistência das matrizes individuais dos especialistas, ou nos processos de incorporação dos resultados, ou mesmo por acumulação de erros de precisão, dentre outras formas de inconsistência (SAATY, 1991). Nesses casos, os resultados obtidos de cada especialista se tornariam ineficazes aos objetivos do modelo, ou seja, de nada ajudariam na tomada de decisão. Seria necessário reiniciar o processo de coleta de novas matrizes individuais, o que por vezes se torna inviável dado a diversas razões, entre as quais, tempo, custos operacionais, desgaste dos especialistas etc.

O que propomos nesse trabalho é a utilização de métodos de pesquisa estocástica conhecidos como Algoritmos Genéticos para criar soluções alternativas nessas situações. Considerando que a matriz do especialista em uma aplicação de Metodologia Multicritério ou de uma técnica de conclave utilizando matriz decisória é muito pouco consistente, nosso objetivo passa a ser criar n soluções (matrizes similares), caso possível, através de uma análise de perturbação de valores da matriz original, quer sejam pequenas perturbações em todos os valores, quer grandes perturbações em alguns poucos valores de modo que satisfaçam o modelo de consistência, possibilitando a geração de uma família de matrizes-solução de forma a permitir ao especialista, nem sempre um profissional ligado às ciências exatas, substituir ou até mesmo complementar sua alternativa.

\section{Metodologia matricial}

Uma Metodologia Multicritério de Apoio à Decisão, de fácil aplicação e muito utilizada com êxito no caso específico de matrizes decisórias ou de apoio à decisão é apresentada em Costa (1999), baseada no trabalho desenvolvido por Crawford e Williams (1985). Considerando-se os atributos $E_{1}, E_{2}, \ldots, E_{n}$, que contribuem para a consecução de um dado objetivo, basicamente, as metodologias aqui em questão se fundamentam em uma comparação dois a dois da importância relativa entre os pares de atributos. Dessa forma, cada especialista poderá construir uma Matriz de Julgamento individual, do tipo $\mathrm{A}=\left[\mathrm{a}_{\mathrm{ij}}\right] \mathrm{nxn}$, onde $\mathrm{a}_{\mathrm{ij}}$ representa a importância relativa do atributo $E_{i}$ em relação ao atributo $E_{j}$, de modo que $a_{i j}>1$, se e somente se $E_{i}$ for mais importante que $E_{j}$ e, $a_{i j}$ $=1 / \mathrm{a}_{\mathrm{ji}}$ para qualquer par $(\mathrm{i}, \mathrm{j})$.

Depois de colhidas as matrizes individuais, uma alternativa para conjugar as informações fornecidas pelos diferentes avaliadores é dada pela média aritmética das matrizes individuais, ou seja, construímos a matriz $\mathrm{A}$, da forma $\mathrm{a}_{\mathrm{ij}}=1 / \mathrm{m} \sum \mathrm{a}_{\mathrm{ijk}}$, onde $\mathrm{m}$ é o número de avaliadores e $\mathrm{a}_{\mathrm{ijk}}$ é o valor proposto para aij pelo k-ésimo especialista consultado. Ocorre que os $\mathrm{a}_{\mathrm{ij}}$ médios já não apresentam as propriedades iniciais (reciprocidade e transitividade). Para resolver esta questão, sugere-se a construção de uma nova matriz (Matriz Média Geométrica), que será formada a partir 
da equação $c_{i j}=v_{i} / v_{j}$, onde cada $v_{i}=\prod a_{i j}{ }^{1 / n}, i=1,2, \ldots, n$, ou seja, $v_{i}$ é a média geométrica dos $a_{i j}$.

Então, satisfazendo as propriedades citadas anteriormente chegamos a uma distribuição de pesos por atributos onde $\mathrm{v}_{1}$ é o peso indicativo ao atributo $\mathrm{E}_{1}, \mathrm{v}_{2}$ é o peso indicativo ao atributo $\mathrm{E}_{2}$, e sucessivamente, de modo que $\mathrm{v}_{\mathrm{i}}$ é o peso indicativo ao atributo $\mathrm{E}_{\mathrm{i}}$. De uma forma geral, de posse dos atributos hierarquicamente distribuídos, o que nos interessa é que as propriedades básicas da matriz simétrica sejam mantidas (também a consistência da matriz resultado final) e, de posse de (n-1) comparações paritárias tenhamos condições de deduzir as demais.

\section{Inconsistência matricial}

Como já dissemos, devido às incertezas, a consistência de qualquer tipo de medida não pode ser garantida. Todas as medidas, mesmo aquelas que fazem uso de instrumentos, por mais sofisticados que estes sejam, estão sujeitas a erros. Um efeito sério e danoso desses erros é que podem, muitas das vezes, levar a conclusões inconsistentes. Mas a consistência perfeita na medida, mesmo com os instrumentos mais aprimorados, é difícil de ser encontrada na prática; então, o que se torna necessário é de um método capaz de avaliar a importância dessa precisão em um problema específico. No nosso caso, o que chamamos inconsistência é uma violação da proporcionalidade, que pode vez por outra significar violação da transitividade. Segundo Saaty (1991), a consistência de uma matriz recíproca e positiva é equivalente a que seu autovalor máximo (GRAYBILL, 1983) seja igual ao número de atributos envolvidos no modelo, ou seja, quanto mais próximo o autovalor máximo estiver do número de atributos do modelo, maior será a coerência dos julgamentos, assim é de se esperar que, numa situação ideal: $\lambda_{\text {máx }}=\mathrm{n}$, onde $\lambda_{\text {máx }}$ é autovalor máximo e $\mathrm{n}$ é número de atributos do modelo.

Também é possível estimar um desvio de consistência pelo resultado da divisão do valor de $\left(\lambda_{\text {máx }}-\mathrm{n}\right)$ por $(\mathrm{n}-1)$. Alguns autores (ver DIAS et al, 1996) preferem utilizar o módulo da diferença entre o número de atributos envolvidos no modelo e o autovalor máximo, ou seja, $\left|\mathrm{n}-\lambda_{\text {máx }}\right|$. De qualquer modo, segundo Saaty (1991) a medida da má consistência poderá ser estimada quando compararmos o valor $\left(\lambda_{\text {máx }}-n\right)$ / $(n-1)$, que chamamos Índice de Consistência (IC), com valores escolhidos de julgamentos aleatórios e seus recíprocos correspondentes, nas posições reversas de uma matriz de mesmo tamanho, segundo testes realizados no laboratório de Oak Ridge e, posteriormente Wharton, com matrizes recíprocas geradas randomicamente (ver SAATY, 1991). A essa medida dá-se o nome Razão de Consistência, de tal forma que RC = IC / IR, onde IR é o Índice (de Consistência) Randômico de uma matriz recíproca e positiva gerada randomicamente e, deve variar de acordo com a ordem de cada matriz. Quanto mais próximo de zero for essa razão, mais consistente estará a matriz, entretanto, é razoável que se essa razão for menor que 0,10 , podemos considerar boa a consistência. Uma justificativa matemática para que o valor da Razão de Consistência (RC) deva ser satisfatório caso menor que 0,10 pode ser encontrada em Vargas (1982).

Entretanto, pode ocorrer que a matriz final, embora representativa da opinião dos especialistas, seja pouco consistente. Isto pode vir a ocorrer devido a falhas na consistência das matrizes individuais, ou mesmo por acumulação de erros. Mais precisamente, estamos nos referindo a matrizes cuja razão de consistência seja superior a 0,10 . Nesses casos, os resultados obtidos de cada especialista se tornariam ineficazes aos objetivos do método, de nada ajudariam na tomada da decisão. Seria necessário reiniciar o processo de coleta de matrizes, o que por vezes se torna inviável dado a razões de tempo, custos operacionais, desgaste dos especialistas. Algumas tentativas recentes de detecção e análise da inconsistência em matrizes podem ser observadas em Pinho et al (1996) e Bramont (1996). Nos dois casos, os autores mostram meios analisadores da inconsistência dirigidos ao método AHP, desenvolvido por Saaty (1991), com respeito à utilização de matrizes no processo de decisão. 


\section{Algoritmos genéticos}

Os Algoritmos Genéticos (HOLLAND, 1975) derivam do modelo de evolução que ocorre na natureza, no qual numa população, cada indivíduo possui um cromossomo composto de bits e são modelos computacionais de busca probabilística que imitam a evolução natural das espécies, combinando os conceitos de adaptação seletiva e sobrevivência dos indivíduos mais capazes. Compreendem um conjunto de indivíduos (população), e um conjunto de operadores genéticos que atua sobre essa população. São computacionalmente simples e poderosos na busca de soluções ótimas, não sendo limitados por restrições tais como considerações sobre continuidade, existência de derivadas e unimodalidade.

Comparando com os tradicionais métodos de otimização, os Algoritmos Genéticos apresentam vantagens tais como: robustez, paralelismo natural, independência, simplicidade e integração (DORNELLAS, 1997). Uma vantagem adicional é a oportunidade de modelar problemas de otimização para os quais não existe uma função objetivo explícita, ou não se pode apresentar numa função matemática, sendo necessário um modelo de simulação para avaliar o desempenho da solução candidata. Uma dificuldade que pode surgir na aplicação dos Algoritmos Genéticos é a definição de uma regra de parada. A solução ideal seria aquela na qual o algoritmo pára sempre que a solução ótima é atingida. As regras de paradas usuais são número máximo de gerações ou um limite de tempo de processamento. Uma alternativa é parar o algoritmo sempre que não exista aperfeiçoamento substancial após algumas gerações.

Ainda que sejam inerentemente aleatórios, os Algoritmos Genéticos utilizam, de forma bastante eficiente, as informações históricas contidas em seu código para a partir daí sugerir novas soluções que melhoram o resultado final do problema. Por isso, são classificados como algoritmos de inteligência emergente (ANGELINE, 1993). Holland (1975) demonstrou que os Algoritmos Genéticos percorrem o espaço amostral em ordem cúbica $\left(\mathrm{n}^{3}\right)$, ou seja, enquanto as iterações crescem em ordem n (o que é computacionalmente interessante) a quantidade de pontos percorridos no espaço amostral cresce em ordem $\mathrm{n}^{3}$. Isto é particularmente interessante em casos de problemas extremamente onerosos em tempo. Aguiar e Costa (1997) mostram uma aplicação na qual uma pesquisa exaustiva (varredura completa do espaço amostral) que levaria em média 50 horas pode ser realizada por uma implementação de Algoritmos Genéticos em aproximadamente 38 segundos.

O Algoritmo Genético consiste num ciclo com os seguintes estágios: criação de uma população de soluções potenciais codificadas (indivíduos); avaliação desses indivíduos; seleção dos indivíduos mais capazes; criação da nova população através de manipulações genéticas. Como a Evolução Natural, trabalham com soluções, que nada mais são do que strings de bits, que codificam parâmetros para o problema. Tais soluções são chamadas de indivíduos, e o conjunto delas é chamado de população. O tamanho da população afeta a eficiência do algoritmo. Cada iteração do algoritmo é chamada de geração, e no presente trabalho os termos serão livremente misturados e confundidos entre si, uma vez que representam as mesmas coisas.

Os Algoritmos Genéticos funcionam a partir de uma função de avaliação (ou aptidão) chamada Função Fitness e de uma série de operadores (seleção, reprodução, mutação e substituição). Essa função é uma métrica que diagnostica o quão adaptado é o indivíduo, em outras palavras, o quão boa é a solução. O operador Seleção escolhe os indivíduos que se reproduzirão; no operador Reprodução, ocorre a combinação de duas ou mais soluções para a geração de outras novas; o operador Mutação nada mais é do que uma alteração de bits visando restaurar a diversidade da população e o operador Substituição é o responsável por substituir soluções antigas pelas remanescentes dos processos de reprodução e de mutação. Maiores considerações sobre o assunto e suas aplicações, ver Gen e Cheng (2000).

\section{O modelo}

Partindo-se de uma matriz de uma aplicação de Metodologia Multicritério com RC maior que $10 \%$, o objetivo do sistema passa a ser gerar n soluções (matrizes similares), caso possível, cuja 
referida razão esteja abaixo desse índice ou, em casos específicos, pelo menos próximos o bastante desse valor, de forma a não influenciar determinantemente a má consistência da matriz opinião dos especialistas (matriz final) e possibilitando, assim, ao(s) decisor(es) ou especialista(s) opções de matrizes muito próximas da original, só que consistentes (ou mais consistentes que a original). A idéia é considerarmos, para esse modelo, matrizes com valores de inconsistência bastante variáveis, desde próximos de $10 \%$, até distantes suficientes desse valor, desde que permitam soluções de razoável proximidade à original.

Considerando uma matriz fornecida pelo resultado de um processo decisório ou por um especialista em particular, o programa analisa inicialmente a consistência da matriz. São calculados o autovalor máximo, o índice e a razão de consistência. Caso essa razão seja superior a 10\%, a matriz é considerada inconsistente e o algoritmo gera aleatoriamente uma série de matrizes de pequenas perturbações nos valores (evitando-se a diagonal unitária) e a partir desta gera uma população inicial. Essa população passa pelos operadores (seleção, reprodução, mutação e substituição) gerando nova população de matrizes, as quais terão sua função de aptidão analisada continuamente. $\mathrm{O}$ processo se encerra quando atingir o ótimo, ou seja, quando for possível gerar matriz(es) consideradas de boa consistência (RC inferior a 10\%). Dessa forma, a finalidade do sistema é: processar uma matriz de entrada através de Algoritmo Genético, de forma a obter uma ou mais matrizes de saída com as características desejadas, em função de sua razão de consistência (RC) e de seu grau de reciprocidade.

Para funcionar com o Algoritmo Genético, uma matriz deve ser codificada como uma série de bits. Optou-se por codificar perturbações em torno dos elementos da matriz, de forma que o numero de bits necessários para codificar uma matriz é dado pelo seu número de elementos multiplicado pelo numero de bits necessário para realizar as perturbações nos seus elementos. Isto reduz significativamente o numero de bits resultantes da codificação de uma matriz. $\mathrm{O}$ número de bits a serem usados para a codificação e a amplitude máxima (range) para as perturbações nos elementos das matrizes é parametrizável. Cada indivíduo de uma dada geração é recomposto na forma matricial. Então, sua razão de consistência e seu grau de não reciprocidade são calculados. A não reciprocidade da matriz corresponde à medida do maior afastamento que um elemento da matriz possui em relação à condição ideal de reciprocidade.

A seguir são analisados os passos desse processo, apresentando de forma adequada os operadores, além das condições de inicio e fim, comentários, e uma simulação de funcionamento. Vale citar que o modelo computacional funciona em linguagem $\mathrm{C}++$.

Inicialização: a população inicial de $\mathrm{N}$ indivíduos é gerada aleatoriamente. Essa questão é parametrizável, podendo variar a cada exemplo, como de fato ocorre e, com matrizes compostas de valores com pequenas perturbações, que deverão evoluir naturalmente, para posteriormente serem acrescidas a matriz inicial, para serem avaliadas quanto ao grau de consistência. São matrizes quadráticas da forma $\mathrm{n} \times \mathrm{n}$, onde $\mathrm{n}$ varia de 2 a 9 . Os limites impostos às dimensões da matriz ( 2 a 9), seguem orientação de Saaty (1991). É importante ressaltar que, como no caso biológico, não há evolução sem variedade, quanto mais diversos forem os indivíduos da população inicial mais facilmente o modelo convergirá para a solução.

Avaliação: os Algoritmos Genéticos necessitam da informação de uma função de aptidão para cada membro da população. Esta função dá a cada indivíduo a medida de adequação a situação estudada. Optou-se inicialmente em utilizar a chamada Razão de Consistência desenvolvida por Saaty (1991). Dessa forma a chamada Função Fitness seria assim representada: Função Fitness $=1$ - RC. Como o valor de RC varia de zero a um $(0,1)$, então a função jamais seria negativa (como requer o procedimento do algoritmo) e, quanto maior fosse mais apto estaria o indivíduo (solução). Entretanto, foi verificado que o simples fato do RC ser significativamente baixo, não é bastante para garantir as condições (propriedades) da matriz recíproca e positiva. Dessa forma, a função sofreu uma alteração, que podemos chamar de restrição, de modo que não basta que o RC seja consideravelmente baixo (menor que 10\%), mas também é condição necessária que a porcentagem de valores da matriz solução que desrespeitam a condição de anti-simetria, seja também inferior a $10 \%$. Assim a nova função passa a ser: Função Fitness $=1-(\mathrm{RC}+\mathrm{NR}) \operatorname{com} \mathrm{NR}=\mid 1-(\mathrm{Tr}+$ aij $\mathrm{x}$ 
aji $) \mid$, onde RC = Razão de Consistência, NR = Não Reciprocidade, $\mathrm{Tr}=$ Taxa de Tolerância, aij = elemento da linha i e coluna $\mathrm{j}$. A não reciprocidade (NR) serve como um gatilho a partir do qual a não reciprocidade é computada na Função de Aptidão (Fitness). Para valores de não reciprocidade inferiores ao limite estabelecido, a Função Fitness volta a ser avaliada da forma inicial. Tal condição de gatilho permite desconsiderar por completo a influência de uma não reciprocidade tolerável na Função de Aptidão, de forma a permitir que o algoritmo avalie os indivíduos em termos de sua razão de consistência até um limite tolerável de não reciprocidade. Uma matriz de saída é considerada como solução caso sua Função de Aptidão avaliada pelo algoritmo esteja acima do valor de limite estabelecido pelo usuário.

Seleção: é o operador que seleciona os indivíduos que estão aptos a se reproduzir. Como mecanismo de Seleção foi usado o método de Ranking with Roulette Wheel, desenvolvido por Aguiar e Costa (1997), e que trabalha da seguinte forma: a cada indivíduo é associado um número ou ranking $\mathrm{R}_{\mathrm{i}}$, de forma que: $\mathrm{R}_{\mathrm{i}}=\left(\mathrm{N}_{\mathrm{i}} / \mathrm{N}\right)^{2}$, onde $\mathrm{N}_{\mathrm{i}}$ é o número de indivíduos com Fitness menor que o do indivíduo i e $\mathrm{N}$ é o número total de indivíduos na população. Após a definição desses números, aplica-se o método tradicional de Roulette Wheel, que consiste no sorteio, respeitando a probabilidade de cada indivíduo ser sorteado.

Cruzamento: no operador Cruzamento (ou Reprodução), foi utilizado o Single Point (um único ponto), e a taxa de reprodução utilizada ou probabilidade de cruzamento foi de $10 \%$. Entretanto tanto no caso do tipo de cruzamento quanto no caso da taxa, os valores escolhidos podem ser arbitrados pelo operador do sistema, visto que o modelo é todo parametrizável.

Mutação: o processo de mutação serve para garantir a diversidade dos indivíduos da população. A questão é definir então com qual freqüência isso deva ocorrer. A probabilidade ou taxa de mutação utilizada foi de $1 \%$. Como no caso da taxa de reprodução esse valor pode ser a qualquer momento alterado a critério do usuário.

Condições de Parada: a regra de parada do algoritmo utilizada foi, quando encontrar pelo menos uma solução ou indivíduo que tenha Função Fitness inferior a 10\%, o algoritmo pode parar. Dessa forma, não se limita o número de soluções a serem encontradas, cabendo ao usuário essa função. Entretanto, como todo modelo que permite intervenção do usuário, durante o processo, o algoritmo pode ser parado a qualquer instante, desde que seja de interesse do usuário. É óbvio que, caso seja de interesse do usuário, o modelo pode estabelecer um limite de tempo para encontrar uma ou mais soluções.

Descrição do Ambiente do Sistema: o sistema foi desenvolvido em linguagem de programação $\mathrm{C}++$, usando biblioteca padrão, de forma a permitir elevado grau de portabilidade. Foi usado o ambiente integrado de desenvolvimento Bloodshed Dev-C++, versão 4, compilador Mingw compiler 2.95.2-1 e debugger GNU Debugger 4.18 (GDB).

Pré-requisitos de Entrada: como requisitos, a matriz de entrada deve ser quadrada e diagonal. O sistema lê a matriz de um arquivo texto, no formato ascii. A primeira linha deve conter a dimensão da matriz. As linhas seguintes devem conter cada uma linha da matriz, sendo os elementos separados por espaços em branco.

Saída do Sistema: o sistema mostra na saída-padrão os resultados do processamento.

Como observação, para flexibilizar e auxiliar no processo de análise e convergência de resultados, o sistema mantém parametrizados alguns itens que têm influência direta nas características de processamento. Cada parâmetro tem um valor "default" pré-definido internamente. Opcionalmente, pode-se especificar o valor através da linha de comando tais como a quantidade de indivíduos da população gerada pelo Algoritmo, a quantidade mínima de indivíduos aptos para caracterizar uma solução, o valor mínimo acima do qual um indivíduo é considerado como apto, a amplitude da excursão máxima para a perturbação em torno do valor de um elemento da matriz, o valor a partir do qual é computada a não reciprocidade no Fitness e o numero de bits para codificação das perturbações. 


\section{Exemplo}

Para serem utilizadas como testes no modelo, foram geradas matrizes aleatórias com diagonal unitárias com variadas ordens de grandeza. Entretanto para constar como parte documentada da evolução do modelo, optou-se por utilizar matrizes extraídas de situações já estudadas e citar fontes. A fonte é Saaty (1991), devido a ser este justamente o estudo mais detalhado do assunto de inconsistência matricial e, as matrizes variam em ordem e grau de inconsistência. Segue um quadro (Figura 1) com as características das matrizes (Razões de Consistência inicial e final de matrizes testadas como exemplo).

Figura 1: Características das Matrizes

\begin{tabular}{|c|c|c|c|c|c|c|}
\hline Matriz & Ordem & População & RC inicial & Gerações & RC final & Fitness \\
\hline 1 & 3 & 100 & 0.116884 & 1380 & 0.0737133 & 0.901138 \\
\hline 2 & 6 & 100 & 0.238381 & 2262 & 0.0997367 & 0.900263 \\
\hline 3 & 4 & 500 & 0.532033 & 291 & 0.0905882 & 0.909412 \\
\hline 4 & 6 & 500 & 0.127419 & 117 & 0.0182739 & 0.981726 \\
\hline & & & & & 0.0920225 & 0.907978 \\
\hline 5 & 3 & 100 & 0.116884 & 492 & 0.0846125 & 0.915388 \\
\cline { 5 - 7 } & & & & & 0.0541502 & 0.903442 \\
\hline
\end{tabular}

As respostas obtidas foram bastante satisfatórias, dentro dos parâmetros envolvidos, respeitando as condições do modelo. Repare que, em alguns casos, o tamanho da população é pequeno e, dessa forma, o sistema converge rapidamente. Na matriz 2, a resposta encontrada pelo sistema é interessante, considerando que o RC da matriz inicial é relativamente alto (note que o número de gerações é o mais alto). Especificamente, no caso da matriz 3, o alto valor do RC inicial dificultou muito o resultado final e, isso sugere que altos valores de RC inicial merecem tratamento especial. $O$ caso da matriz 5 é, na realidade, uma variação da matriz 1, alterando-se o critério de parada de uma para três soluções. As matrizes respostas encontradas pelo sistema são bastante satisfatórias e os valores de RC apresentam pequenas e não significativas variações. Nos casos das matrizes 3 e 4, foi aumentado de forma intencional o tamanho da população inicial, o que em geral resultaria em soluções mais adaptadas.

\section{Conclusões}

O objetivo principal desse trabalho é oferecer uma proposta para solução do problema de inconsistência de matrizes decisórias. A abordagem utilizada são os Algoritmos Genéticos, que são apresentados tanto do ponto de vista teórico quanto do prático (o modelo desenvolvido). No que diz respeito à inconsistência matricial numa análise de decisão, é consenso, e o presente trabalho tenta comprovar, que a inconsistência deve ser tratada, visando minimizar os prejuízos do processo decisório (facilitar a tomada de decisão). Também ressaltamos que a maneira mais viável de resolver esta questão deve ser a utilização de sistemas de apoio à decisão.

A importância dos Algoritmos Genéticos no tema se insere nas diversas vantagens que esse tipo de método computacional oferece, entre as quais a de podermos gerar mais de uma solução para cada matriz com problema de inconsistência, permitindo que o decisor tenha um maior número de opções para substituir a matriz inicial. Esse assunto pode, por um lado parecer um complicador, visto que em algumas circunstâncias, o tomador de decisão pode não se mostrar interessado em escolher uma entre várias soluções próximas da otimalidade. Entretanto isso se torna bastante simples de resolver bastando recalibrar o algoritmo (modelo) para oferecer somente uma solução 
para cada problema (matriz).

Quanto ao modelo de algoritmo, ocorreu boa resposta, sem a necessidade de intervenção do usuário, não havendo variações da taxa de mutação e de cruzamento, embora essas situações sejam muito comuns em aplicações de Algoritmos Genéticos e, de forma alguma diminuam a validade e propriedade do sistema. Podemos observar ainda que ocorreu variação da taxa de reciprocidade, de acordo com o exemplo, visando, em geral, não fugir por demais da realidade (características) da matriz inicial. Uma preocupação que devemos ter quando da utilização de modelos computacionais, é o tempo de computação, um fator onerante na solução do problema. Nesse caso, foi relativamente pequeno e, considerando as dimensões do problema proposto, muito próximo do esperado, o que aponta para mais uma vantagem.

Importante ressaltar que os modelos utilizados como exemplos foram todos extraídos de situações reais, de casos estudados e publicados e, que na maioria desses exemplos, considera-se satisfatório que a condição de reciprocidade seja respeitada, dando-se menor grau de importância à condição de transitividade. De fato, alguns autores consideram que a idéia de intransitividade entre preferências pode ser vista como um fenômeno natural, e não como uma conseqüência de erros de julgamentos

Outra questão notada e bastante relevante é a da definição de critérios mais acurados para diagnosticar a consistência das matrizes, que complementem a utilização da Razão de Consistência proposta por Saaty (1991).

\begin{abstract}
This work presents a proposition to solve the problem of inconsistency in decision matrices using genetic algorithms. The Genetic Algorithms are probabilistic search computer models which are based on the mechanics of natural selection and natural genetics, combining the concepts of selective adaptation and survival of the fittest. They are considered to be a powerful technique of stochastic optimization and, probably the most important evolutionary computer techniques. Its application to the decision matrices case allows the detection of inconsistent matrices, while offers alternative solutions to the decision-maker.
\end{abstract}

Keywords: Decision; Inconsistent Matrices; Genetic Algorithms.

\title{
Referências
}

AGUIAR, E.; COSTA, J. F. S. Uma implementação de pesquisa estocástica utilizando algoritmos evolutivos. Cadernos do IME, Rio de Janeiro, v. 1, 1997.

ANGELINE, P. J. Evolutionary algorithms and emergent intelligence. Dissertation, The Ohio State University, USA. 1993.

BRAMONT, B. P. P. Consideração de incertezas nas estimativas de pesos dos critérios em MCDM. Anais do XVI ENEGEP e II Congresso Internacional de Engenharia Industrial, Piracicaba, SP. 1996.

COSEnZA, O. N., SOUZA, C.G. Manual de técnicas de conclaves. Rio de Janeiro, DNER. 1986.

COSTA, J.F.S. Uma aplicação da metodologia multicritério na qualidade do ensino. Anais do XIX ENEGEP-IV Congresso Internacional de Engenharia Industrial, Rio de Janeiro, RJ. 1999.

CRAWFORD, G., WILLIAMS, C. The analysis of subjetive judgement matrices. The Rand Corporation R- 2572-1AF, USA. 1985.

DIAS, L.M.C., ALMEIDA, L.M.A.T., CLÍMACO, J.C.N. Apoio multicritério à decisão. Portugal, Universidade de Coimbra. 1996.

DORNELLAS, C.R.R. Otimização dos despachos reativos utilizando algoritmos genéticos. Tese de Mestrado, COPPE - UFRJ, Rio de Janeiro. 1997. 
GEN, M., CHENG, R. Genetic algorithms $\square$ engineering optimization. Wiley, NY, USA. 2000.

GRAYBILL, F. A. Matrices with applications in statistics. California, USA: Wadsworth Inc., 1983.

HOLLAND J. Adaptation in natural and artificial systems. The Univ of Michigan Press, Michigan, USA. 1975.

PINHO, F.A., MONTEVECHI, J.A.B., PAMPLONA, E.O. Um modelo computacional baseado no método AHP para análise multicriterial de decisão. Anais do XVI ENEGEP e II Congresso Internacional de Engenharia Industrial, Piracicaba, SP. 1996.

ROY, B. Methodologie multicritere d'aide á la decision. Paris, Economica. 1985.

WANDERLEY, A. J. M., COSTA, J. F. S. Utilização de matrizes em problemas de tomada de decisão estratégica. Revista UNIANDRADE, Curitiba, Ed.1, Vol. 3, ano II. 2002.

SAATY, T. L. Método de análise hierárquica. 2. ed. São Paulo: Makrom Books, 1991.

VARGAS, L.G. Reciprocal matrices with random coefficients. Math. Model, 3, 69-81, USA. 1982. 